\title{
Evaluation of the temporal effect to the peak tailing in flow injection analysis
}

\author{
Su-Cheng Pai* \\ Division of Marine Chemistry, Institute of Oceanography, National Taiwan University, P.O. Box 23-13, Taipei, Taiwan
}

Received 7 September 2001; received in revised form 3 January 2002; accepted 8 January 2002

\begin{abstract}
The deformation of a flow injection analysis peak from a Gaussian shape has two components: spatial and temporal. The former is mainly attributed to the Poisseulie effect in the tubular flow, whereas the latter is related to the observing position (of a fixed detector) at which signal is measured. The combination of the two makes a skewed peak track on the recorder chart. Therefore, an observed peak may imply a substantial fraction of a "false" tail due to the effect of non-simultaneous detection. An expanding-Gaussian model is proposed to simulate the purely temporal effect, and the asymmetric factors were compared with that of the experimental peak shapes. In most cases the peak deformation occurring in flow injection analysis should be regarded as "temporal". The contribution of the spatial effects (Poisseulie profile and others) might not be as significant as it was thought previously. (c) 2002 Elsevier Science B.V. All rights reserved.
\end{abstract}

Keywords: Peak tailing; Flow injection analysis; Temporal effect

\section{Introduction}

Many chemical instruments give output signals as peaks. In chromatography the peak is generally symmetrical or Gaussian-like, but for flow injection analysis (FIA) it is frequently skewed with a tail. The tailing phenomenon, important for optimizing instrumental conditions, has drawn intensive attention since the technique was introduced in 1975 [1]. A question is raised: why is the FIA peak asymmetrical? One may seek assistance from existing chromatographic theories as both techniques share very much the same physical principles [2]. Several chromatographic models are helpful in this respect including the progressively calculated plate [3] or

*Tel.: + 886-2-2362-7358; fax: +886-2-2363-2912.

E-mail address: scpai@ccms.ntu.edu.tw (S.-C. Pai). tank-in-series model [4], the stochastic statistical model [5], and the solutions of the partial differential equations for mass transfer kinetics along a column [6-8]. However, after a numerical comparison Golshan-Shirazi and Guiochon [9] concluded that all chromatographic models can be approximated by a Gaussian profile even when the column efficiency is low. Although each model does give an asymmetrical peak shape at the initial stage, the skewness is considered "insignificant", far from enough to justify the observations commonly seen in FIA work.

Further explanations become complex. The concept of "non-linear isotherms" have been used in adsorption chromatography to derive non-symmetrical peaks [10], but it surely cannot be valid for FIA due to the lack of a stationary phase. Instead, the physical models derived from Fick's Law [11-15], which is based on the physical dispersion-diffusion 
behaviors of a tubular flow, have been more favored. Together with the friction effect against the tube wall, the resultant "Poisseulie" or the "parabolic velocity" profile has been generally adopted as the major source for the peak tailing in flow injection analysis, and the "bow-shape" diagram appears frequently in papers and textbooks $[1,16]$.

\subsection{The observer's position}

One important factor, the observer's effect or the temporal effect, might have been overlooked by FIA analysts. When a shape-changing object passes through a fixed narrow-slit scanning camera, the observer will receive a distorted picture. It relates only to the observer's standing point and the movement of that object, and not to the real appearance of the object at a time. The deformation due to the non-simultaneous detection is spatially "false". An "observed peak shape" implies the "real peak shape" plus "temporal deformation". The concept is not novel in the chromatographic field. Back in the 1950 s, a continuous plate theory given by Glueckhauf [17] has already demonstrated that the peak shape can be distorted to have a tail even when the mass distribution in a column is Gaussian. A worksheet practice of the Glueckauf's model is included in a recent textbook by de Levie [18].

Misinterpretation might start from here: some FIA analysts tend to use experimentally observed peak shape to represent the mass-distribution pattern in the tubing, as they deemed the difference is negligible. Indeed, the mass distribution curve, based on a length coordinate, can be converted to a time scale. For example, the mean residence time or peak appearance time $t_{\mathrm{p}}$ can be calculated from $t_{\mathrm{p}}=$ $\pi r^{2} L / F$ (where $r$ is the tube radius, $L$ the length of the tubing, $F$ the flow-rate) or $t_{\mathrm{p}}=L / u$ ( $u$ the flow speed). This "converted" time coordinate, although having identical scaling to that on the "observer's" time coordinate, is still spatial in nature. Without making a distinction between the two may lead to further confusion when dealing with the peak shape problems.

\section{Theoretical}

A diagram (Fig. 1) is presented to distinguish the
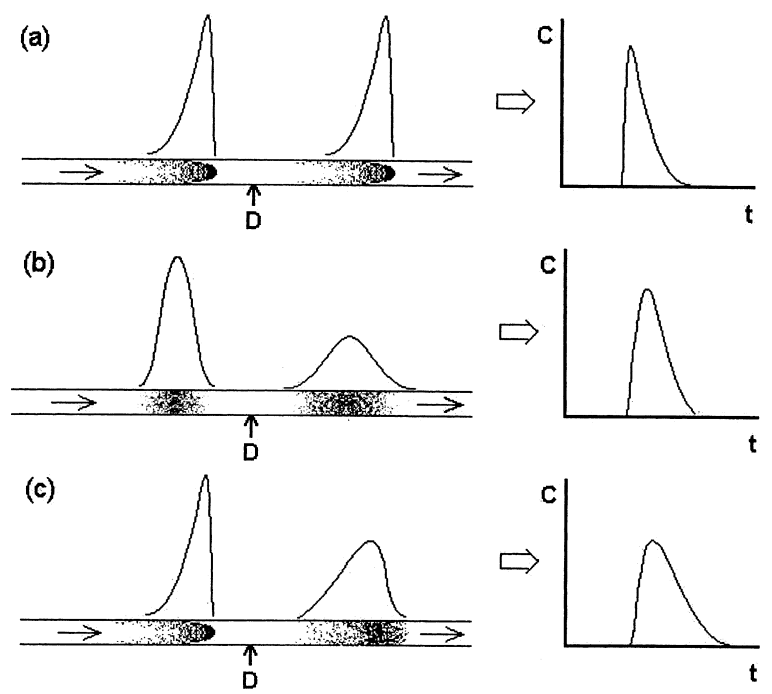

Fig. 1. Diagrams illustrating the possible relationships between the mass profile (left) and recorded peak shape (right). (a) Assuming that the mass distribution in the tubular channel is asymmetrical due to the Poisseulie effect, and that the pattern is unchanged during its passage through the detector at position D, the recorder will give peak shape exactly like the mass distribution peak. The deformation of peak is purely spatial. (b) Assuming the mass distribution is symmetrical throughout but the sample zone is expanding while moving, the detector will record a steeper peak front and a prolonged peak tail. The deformation of peak is purely temporal. (c) In real situation the skewed peak image might attribute to the combination of both.

"spatial profile" and "temporal deformation". They are separated on a hypothetical basis. In the former case (Fig. 1a), the injected sample section moves faster at the center axis than near the tube wall, leading to an asymmetric distribution pattern. If the pattern remains unchanged during its passage through a detector, a skewed shape is recorded. The image reflects a purely spatial pattern. In the latter case (Fig. 1b), if the injected sample section has already become a Gaussian-like pattern soon after leaving the injector, and it is still broadening with time as it travels, the sensor encounters first a steeper front and then records a prolonged tail when the main peak passes over. The deformation of the observed peak is purely temporal.

In a real situation, the skewness of a peak should refer to the combination of the two processes (see Fig. 1c). However, since one can always derive the asymmetrical parabolic laminar flow by equations, only the spatial effect has been emphasized by FIA 
modelists. The temporal effect, sounds insignificant and difficult to verify experimentally, has been usually ignored.

\subsection{Mathematical approach}

For laboratory applicants, the mathematical approaches are of value if they can be simple enough to be practiced on a computer spreadsheet (e.g., Excel). The most successful one would be the exponentially modified Gaussian function (EMG) [19-22]. It is based on the fact that all complicated models converge to a Gaussian function, and the degree of deformation decays exponentially with time. Thus, through a convolution process, an EMG curve is generated (in Gaussian form plus decay function). The EMG function can indeed produce significant asymmetric peak shape even at high theoretical plate numbers. Even though the physical meaning has been questioned by some scientists [9], it has been widely adopted as a reasonable approach in the chromatography field [23].

\subsection{Conceptual approach}

A more simple approach is described in this work namely, the expanding-Gaussian function. It follows the posture of the EMG function that the mass profile in a tubular channel is eventually Gaussian. Instead of converging an exponential function, the Gaussian curve is "transformed" from a spatial axis to a temporal axis. The skewness of the observed peak is generated by the Gaussian curve itself, as it expands while it moves. Therefore, the present model is more a "conceptual" one than a mathematical fitting.

Only two basic equations are involved: a standard Gaussian equation, and one describing the expanding of its standard deviation along the $x$-axis. In FIA, $x$ can be the length $L$ of the tubular channel or time $t$. According to previous theories, the "zone-broadening" of a Gaussian profile can be defined as the expanding of the standard deviation $\sigma_{L}$ of the peak, which is proportional to the square root of the travel distance $L$ at a constant flow speed (or $\sigma_{L}^{2} \propto L$ ):

$\sigma_{L}=\mu_{L} \sqrt{L}$

where $\mu_{L}$ is defined here as an "expanding coefficient" (on $L$ coordinate) of the flow system. (Note that this coefficient, in $\mathrm{cm}^{1 / 2}$ unit, is equivalent to the square root of the theoretical plate height $H_{L}$ in chromatography. It also equals $\sqrt{2 D / u}$ in Fick's law where $D$ is the diffusion coefficient and $u$ the flow speed [23]. The purpose of using of $\mu_{L}$ or later $\mu_{t}$ in this paper is to emphasize the "expanding" nature of the injected sample zone, just for the convenience of FIA readers.)

\subsection{Spatial distribution profile}

Combining Eq. (1) and a standard Gaussian equation and letting the total peak area as $A_{L}$, a symmetric concentration distribution pattern $C(L)$ at a given time is generated and it can be plotted on the $L$ coordinate:

$C(L)=\frac{A_{L}}{\mu_{L} \sqrt{2 \pi L_{\mathrm{p}}}} \mathrm{e}^{-\left(\frac{L-L_{\mathrm{p}}}{\mu_{L} \sqrt{2 L_{\mathrm{p}}}}\right)^{2}}$

where $L_{\mathrm{p}}$ is the position of peak summit (usually refers to the position of the detector on the $L$ coordinate). The curve generated by Eq. (2), noted as the mass distribution curve, cannot be observed temporally by a single fixed detector. It could only be seen if there were "many" detectors set along the channel to detect simultaneously.

It is very easy for one to think that Eq. (2) can be converted into time scale, as $t_{\mathrm{p}}$ is proportional to $L_{\mathrm{p}}\left(t_{\mathrm{p}}=L_{\mathrm{p}} / u\right)$, and $\sigma_{t}^{2} \propto t_{\mathrm{p}} ; A_{L}$ and $\mu_{L}$ can be transformed into temporal terms $A_{t}$ and $\mu_{t}$ correspondingly. Thus, it can be "mistakenly" expressed as:

$C(t)=\frac{A_{t}}{\mu_{t} \sqrt{2 \pi t}} \mathrm{e}^{-\left(\frac{t-t_{\mathrm{p}}}{\mu_{t} \sqrt{2 t_{\mathrm{p}}}}\right)^{2}}$

This Gaussian-type curve, although on a time scale, does not reveal the recorder output, as what the detector receives should be on a "sequential" time axis. Therefore the peak generated by Eq. (3) does not exist and should not be plotted.

\subsection{Temporal convolution}

To project the non-existing image of Eq. (3) from a "simultaneous" time coordinate to a "real" time coordinate is named the "temporal convolution". Since the observation is made at a single point, one should consider the standard deviation of a peak $\left(\sigma_{t}\right)$ is variable at all time $\left(\sigma_{t} \propto \sqrt{t}\right.$ instead of $\left.\sigma_{t} \propto \sqrt{t_{\mathrm{p}}}\right)$. It 
means that the observed curve is composed of many mass-curve segments at various time $t$. Thus, by replacing $t_{\mathrm{p}}$ as $t$ in the square root terms, a "spatialtemporal projection-transformation equation", or simply the "temporally convoluted Gaussian" (TCG) is given:

$C(t)=\frac{A_{t}}{\mu_{t} \sqrt{2 \pi t}} \mathrm{e}^{-\left(\frac{t-t_{\mathrm{p}}}{\mu_{t} \sqrt{2 t}}\right)^{2}}$

The peak generated by this TCG equation is not symmetrical, but it reveals the output on a recorder chart. The temporal deformation fraction of Eq.(4) from a Gaussian profile can be defined as the difference between Eqs. (3) and (4):

$T D(t)=\frac{A_{t}}{\mu_{t} \sqrt{2 \pi t}} \mathrm{e}^{-\left(\frac{t-t_{\mathrm{p}}}{\mu_{t} \sqrt{2 t}}\right)^{2}}-\frac{A_{t}}{\mu_{t} \sqrt{2 \pi t}} \mathrm{e}^{-\left(\frac{t-t_{\mathrm{p}}}{\mu_{t} \sqrt{2 t_{p}}}\right)^{2}}$

where $T D(t)$ is the temporal deformation function. It has three "crossing knots" on the $t$ axis, one near the peak summit, and the rest two at each side of the peak (Fig. 2). The skewness of a peak relates to both

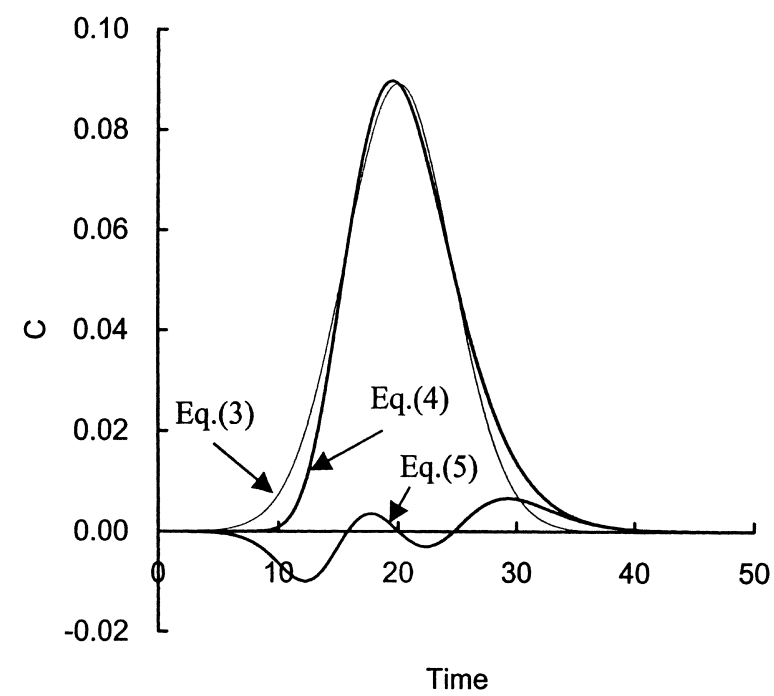

Fig. 2. The temporal deformation function, $T D(t)$ (Eq. (5)) is the difference between a standard Gaussian curve (Eq. (3)) and the TCG curve (Eq. (4)). Assuming $\mu_{t}=1, A_{t}=1$, and $t_{\mathrm{p}}=20$, the curve crosses $t$-axis at $t=15.8,20.0$ and 24.7. The temporal peak $\left(t_{\mathrm{p}}^{*}=19.5, h=0.0898\right)$ appears slightly earlier than that expected $\left(t_{\mathrm{p}}=20, h=0.0892\right)$, with a slightly higher peak height. $t_{\mathrm{p}}$ and $\mu_{t}$, to be significant when $t_{\mathrm{p}}$ is small or $\mu_{t}$ is large, and vice versa.

\subsection{Temporal shift of peak summit}

It is interesting to find that the position of the temporally convoluted peak (note as $t_{\mathrm{p}}^{*}$ ) appears slightly earlier than $t_{\mathrm{p}}$; thus it has an identical peak height to that of the spatial peak at $t_{\mathrm{p}}$ but with a "very" slightly higher peak height at $t_{\mathrm{p}}^{*}$. The small temporal shift $\left(t_{\mathrm{p}}-t_{\mathrm{p}}^{*}\right)$ is related to $\mu_{t}$ but independent to $A_{t}$ or $t_{\mathrm{p}}$. The scale is ca. $0.5 \mathrm{~s}$ when $\mu_{t}=1.0$ $\mathrm{s}^{1 / 2}$ at a constant flow speed, $0.4 \mathrm{~s}$ when $\mu_{t}=0.9$ $\mathrm{s}^{1 / 2}$, and $0.3 \mathrm{~s}$ when $\mu_{t}=0.8 \mathrm{~s}^{1 / 2}$. The expanding coefficient $\mu_{t}$ may vary from system to system. A quick estimation can be made experimentally by measuring the "observed" peak area $A_{t}$, height $h$, and position $t_{\mathrm{p}}^{*}$ :

$\mu_{t} \approx A_{t} / h / \sqrt{2 \pi t_{\mathrm{p}}^{*}}$

In most FIA cases $\mu_{t}$ should be around $1 \mathrm{~s}^{1 / 2}$.

\subsection{Comparison to previous models}

The TCG equation generates promising asymmetric peaks but with no surprise. This conceptual approach, without needing the knowledge of the theoretical plate number, physical diffusion-dispersion or differential equations, leads to a resultant form almost the same as the famous Taylor-Aris series experiment [11,24], also as that derived by Lapidus and Amundson [6] for tubular chromatography. The skewed pattern generated is comparable to that by other sophisticated models as illustrated in the review paper by Golshan-Shirazi and Guiochon [9]. The TCG function is simpler than the EMG function, yet offers similar and reasonable skewed peak shapes.

The question becomes: is the zone broadening symmetrical at the very beginning and throughout, and the observed tail a "false" one due to the temporal effect? Or, is the zone broadening not symmetrical initially, turning to be Gaussian but still viewed as having a temporal tail? Or, does the asymmetric zone broadening accompany with the temporal effect at all time? If both spatial and temporal components co-exist as stated in Fig. 1c, 
analysts would like to know: which is dominating in FIA?

\section{Experimental}

To further verify the temporal effect by experiment, a simplified flow injection system was assembled as shown in Fig. 3. It comprised a peristaltic pump running a distilled water carrier at a constant rate of $4.25 \mathrm{ml} / \mathrm{min}$; a Rheodyne four-way injector having an injection volume of $0.357 \mathrm{ml}$; a Hitachi U-2000 double-beam spectrophotometer installed with a Hellma narrow-bore flow cuvette (light path $1.0 \mathrm{~cm}$, capacity $31 \mu \mathrm{l}$ ); a delay coil that connected between the injector and the detector. A series of delay coil of variable lengths $(20-360 \mathrm{~cm})$ was made using PTFE tube of $0.8 \mathrm{~mm}$ I.D. wound onto a supporting rod. The coiled tubing facilitates mixing in the flow channel [1].

A blue dye solution was used as the testing sample; the undiluted absorbance reading was 0.805 (at $\lambda_{\max }=629 \mathrm{~nm}$ ). Upon the injection of the dye solution (vol. $=0.357 \mathrm{ml}$ ), the recorder was pressed "on" to draw the peak track. Each resultant peak was specified by measuring its peak appearance time $\left(t_{\mathrm{p}}^{*}\right)$, peak height $(h)$, and all those parameters useful to characterize the peak asymmetry [25] (see Fig. 4), i.e., peak widths $\left(W_{0.1}\right.$ and $\left.W_{0.5}\right)$ at $0.1 h$ and $0.5 h$ levels, respectively, components $(a, b, c, d)$ and their corresponding positions $\left(t_{a}, t_{b}, t_{c}, t_{d}\right)$.

\section{Results and discussion}

\subsection{Experimental peaks}

The recorded peak shapes (with delay coil lengths $L=0,20,40,60,80,100,120,140,160,200,260$, $300,360 \mathrm{~cm}$, respectively) obtained by the proposed flow injection system are shown in Fig. 5. The peak information is listed in Table 1. The peak area was estimated to be 4.06 by taking the multiplied value of the initial bandwidth and absorbance of the injected sample. The expanding coefficient $\mu_{t}$ was estimated from Eq. (6), to be $1.01 \pm 0.037 \mathrm{~s}^{1 / 2}$ for this system. The "observed" peak positions were

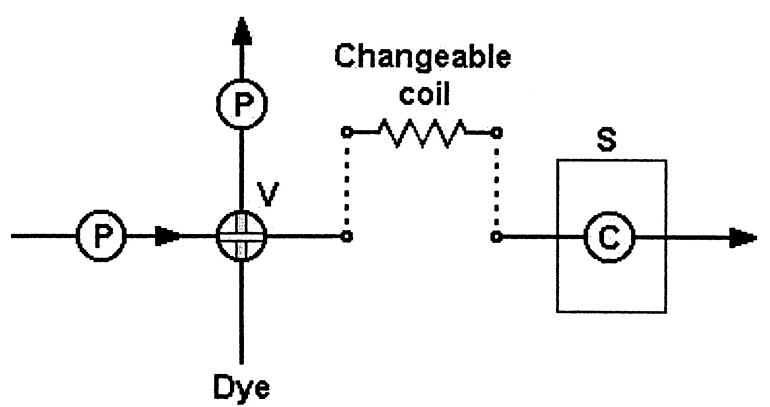

Fig. 3. Layout of the flow injection system used in this study. The delay coil is changeable from 0 to $360 \mathrm{~cm}$ long (made of PTFE tube $0.8 \mathrm{~mm}$ I.D.). P, peristaltic pump; $\mathrm{V}$, injection valve; $\mathrm{S}$, spectrophotometer; C, $1 \mathrm{~cm}$ flow cuvette (capacity $31 \mu \mathrm{l}$ ).

regarded as $t_{\mathrm{p}}^{*}$, whereas the "spatial" position $t_{\mathrm{p}}$ were retrieved by adding to $t_{\mathrm{p}}^{*}$ a temporal shift of 0.5 s.

\subsection{TCG simulations}

By taking the experimental $A_{t}, \mu_{t}$, and corresponding $t_{\mathrm{p}}$ values (Table 1), and putting into the temporally convoluted Gaussian (TCG) equation, a series of peaks was generated (Fig. 6). The asymmetry components $(a$ and $b$ ) were obtained by analyzing the TCG peak at $0.1 h$ level (at $t_{\mathrm{p}}^{*}-a$ and $\left.t_{\mathrm{p}}^{*}+b\right)$. All simulated peak data are listed in Table 2 .

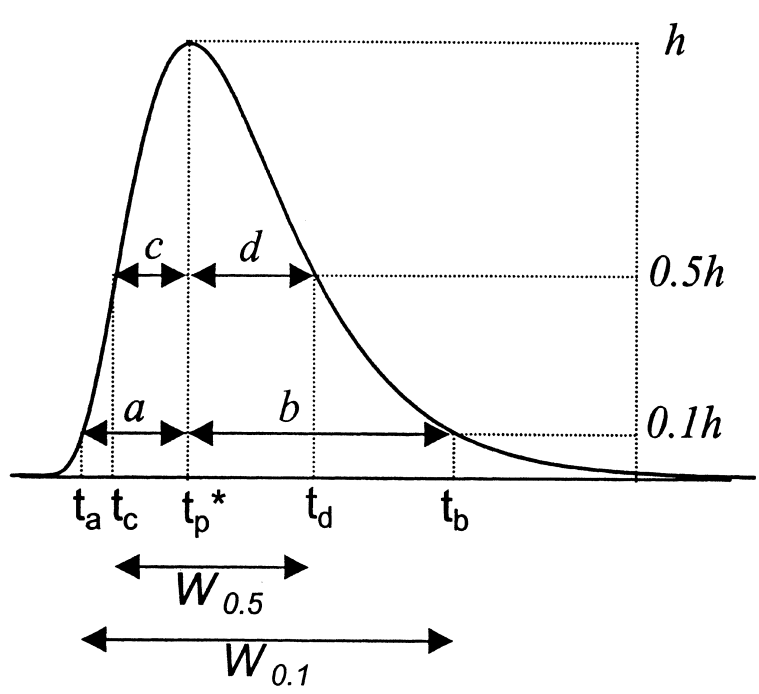

Fig. 4. Parameters of an experimental asymmetrical peak. Asymmetry factor $\left(A_{f}\right)$ is defined by $A_{f}=b / a$. 


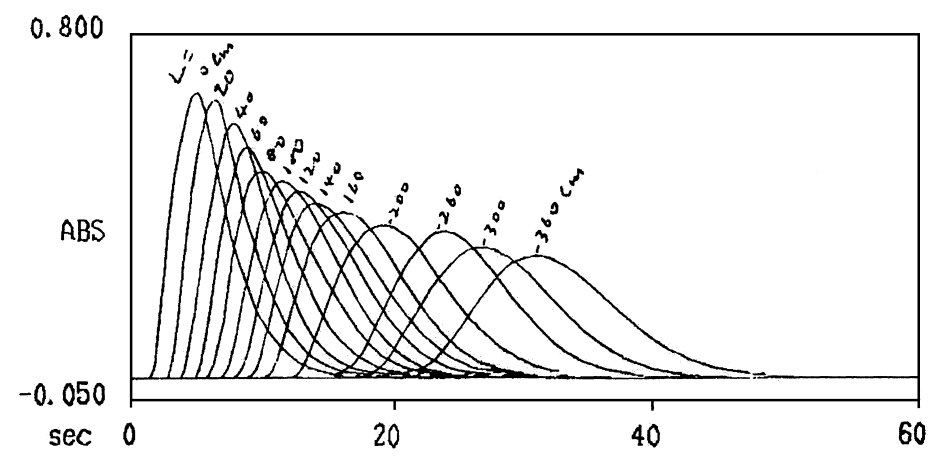

Fig. 5. The peak tracks recorded by a Hitachi U-2000 spectrophotometer for a dye sample injected into the flow injection system with variable delay coil lengths. The injection volume was $0.357 \mathrm{ml}$. The lengths of delay coils were manually marked on the chart.

Table 1

Parameters taken from experimental peaks shown in Fig. 5

\begin{tabular}{|c|c|c|c|c|c|c|c|c|c|c|c|c|c|}
\hline \multirow{2}{*}{$\begin{array}{l}\text { Coil length } \\
L_{\mathrm{c}}(\mathrm{cm})\end{array}$} & \multirow{2}{*}{$\begin{array}{l}\text { Peak height } \\
h \text { (Abs) }\end{array}$} & \multicolumn{5}{|c|}{ Peak position observed (s) } & \multicolumn{2}{|c|}{ Peak width } & \multicolumn{3}{|c|}{ Skewness } & \multirow{2}{*}{$\begin{array}{l}\mu_{t} \text { estimated } \\
\text { by Eq. (6) }\end{array}$} & \multirow{2}{*}{$\begin{array}{l}\text { Spatial } \\
t_{\mathrm{p}}\end{array}$} \\
\hline & & $t_{a}$ & $t_{c}$ & $t_{\mathrm{p}^{*}}$ & $t_{d}$ & $t_{b}$ & $W_{0.5}$ & $W_{0.1}$ & $a$ & $b$ & $A_{f}=b / a$ & & \\
\hline 0 & 0.667 & 2.0 & 3.4 & 5.7 & 8.3 & 11.4 & 4.9 & 9.4 & 3.7 & 5.7 & 1.541 & 1.02 & 6.2 \\
\hline 20 & 0.646 & 3.4 & 4.8 & 7.1 & 10.0 & 13.0 & 5.2 & 9.6 & 3.7 & 5.9 & 1.595 & 0.94 & 7.6 \\
\hline 40 & 0.593 & 4.4 & 5.8 & 8.4 & 11.8 & 15.4 & 6.0 & 11.0 & 4.0 & 7.0 & 1.750 & 0.94 & 8.9 \\
\hline 60 & 0.524 & 5.5 & 7.2 & 9.7 & 13.7 & 17.0 & 6.5 & 11.5 & 4.2 & 7.3 & 1.738 & 0.99 & 10.2 \\
\hline 80 & 0.475 & 6.5 & 8.0 & 10.8 & 15.4 & 18.8 & 7.4 & 12.3 & 4.3 & 8.0 & 1.860 & 1.04 & 11.3 \\
\hline 100 & 0.459 & 7.6 & 9.5 & 12.2 & 17.0 & 20.7 & 7.5 & 13.1 & 4.6 & 8.5 & 1.848 & 1.01 & 12.7 \\
\hline 120 & 0.434 & 8.7 & 10.6 & 13.5 & 18.6 & 22.5 & 8.0 & 13.8 & 4.8 & 9.0 & 1.875 & 1.02 & 14.0 \\
\hline 140 & 0.404 & 9.9 & 11.6 & 14.9 & 20.1 & 24.2 & 8.5 & 14.3 & 5.0 & 9.3 & 1.860 & 1.04 & 15.4 \\
\hline 160 & 0.382 & 11.0 & 13.1 & 16.5 & 22.0 & 26.1 & 8.9 & 15.1 & 5.5 & 9.6 & 1.745 & 1.04 & 17.0 \\
\hline 200 & 0.353 & 13.5 & 15.6 & 19.5 & 25.3 & 29.6 & 9.7 & 16.1 & 6.0 & 10.1 & 1.683 & 1.04 & 20.0 \\
\hline 260 & 0.341 & 17.3 & 20.0 & 24.4 & 30.4 & 35.4 & 10.4 & 18.1 & 7.1 & 11.0 & 1.549 & 0.96 & 24.9 \\
\hline 300 & 0.301 & 19.3 & 22.0 & 27.2 & 33.6 & 39.1 & 11.6 & 19.8 & 7.9 & 11.9 & 1.506 & 1.03 & 27.7 \\
\hline 360 & 0.281 & 23.0 & 26.0 & 31.5 & 38.3 & 43.9 & 12.3 & 20.9 & 8.5 & 12.4 & 1.459 & 1.03 & 32.0 \\
\hline
\end{tabular}

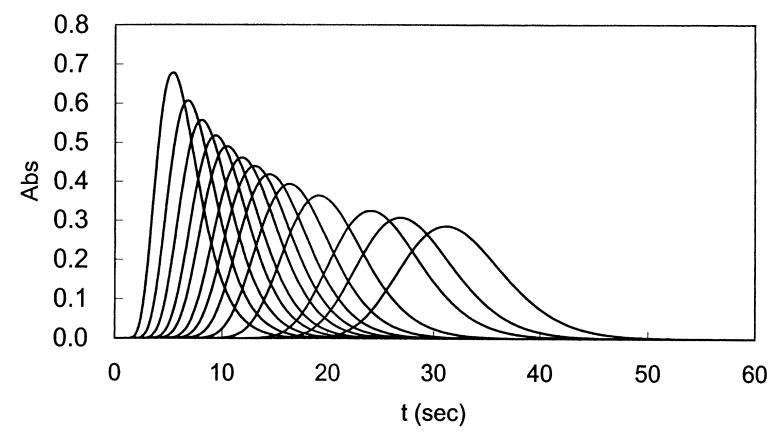

Fig. 6. The observer's FIA peaks generated by Eq. (4) assuming $A_{t}=4.06, \mu_{t}=1.01$, and variable spatial peak positions $\left(t_{\mathrm{p}}=6.2-\right.$ $32 \mathrm{~s}$, respectively, taken from Table 1). All curves were spatially Gaussian before convoluted to the time coordinate. The deformation of the peak shape attributes completely to the temporal effect.
The resemblance of the simulated peak shapes (Fig. 6) to the experimental peaks (Fig. 5) is generally good. They all show characteristics (see Fig. 7) matching the basic FIA rules as stated in textbook by Růžčcka and Hansen [1]: (1) the shape is skewed when the mean residence time $t_{\mathrm{p}}^{*}$ is short; and turns gradually to a more symmetrical-look when $t_{\mathrm{p}}^{*}$ is large; (2) the peak area remains the same at all times; (3) The peak height $h$ decreases with increase of the mean residence time $t_{\mathrm{p}}^{*}$; (4) the peak widths $\left(W_{0.5}, W_{0.1}\right.$ or $\left.a, b\right)$ increase with the square root of $t_{\mathrm{p}}^{*}$. However, discrepancies are obvious when $t_{\mathrm{p}}^{*}$ is very short, say, in the first $10 \mathrm{~s}$ in this work, and they should be regarded as the "sample size" effect. 
Table 2

Simulated peaks generated by Eq. (4) using $A_{t}=4.06, \mu_{t}=1.01$, and corresponding $t_{\mathrm{p}}$ values in Table 1 (also see Fig. 6)

\begin{tabular}{|c|c|c|c|c|c|c|c|c|c|c|c|}
\hline \multirow{2}{*}{$\begin{array}{l}\text { Spatial } \\
t_{\mathrm{p}}(\mathrm{s})\end{array}$} & \multirow{2}{*}{$\begin{array}{l}\text { Peak height } \\
h \text { (Abs) }\end{array}$} & \multicolumn{5}{|c|}{ Peak position calculated (s) } & \multicolumn{2}{|c|}{ Peak width } & \multicolumn{3}{|c|}{ Skewness } \\
\hline & & $t_{a}$ & $t_{c}$ & $t_{\mathrm{p}^{*}}$ & $t_{d}$ & $t_{b}$ & $W_{0.5}$ & $W_{0.1}$ & $a$ & $b$ & $A_{f}=b / a$ \\
\hline 6.2 & 0.657 & 2.48 & 3.58 & 5.70 & 9.17 & 13.38 & 5.59 & 10.90 & 3.22 & 7.68 & 2.385 \\
\hline 7.6 & 0.592 & 3.32 & 4.64 & 7.10 & 10.92 & 15.38 & 6.28 & 12.06 & 3.78 & 8.28 & 2.190 \\
\hline 8.9 & 0.545 & 4.15 & 5.67 & 8.40 & 12.50 & 17.18 & 6.83 & 13.03 & 4.25 & 8.78 & 2.066 \\
\hline 10.2 & 0.508 & 5.00 & 6.71 & 9.70 & 14.06 & 18.95 & 7.35 & 13.95 & 4.70 & 9.25 & 1.968 \\
\hline 11.3 & 0.483 & 5.75 & 7.60 & 10.80 & 15.38 & 20.42 & 7.78 & 14.67 & 5.05 & 9.62 & 1.905 \\
\hline 12.7 & 0.455 & 6.72 & 8.76 & 12.20 & 17.02 & 22.25 & 8.26 & 15.53 & 5.48 & 10.05 & 1.834 \\
\hline 14.0 & 0.433 & 7.66 & 9.85 & 13.50 & 18.54 & 23.94 & 8.69 & 16.28 & 5.84 & 10.44 & 1.788 \\
\hline 15.4 & 0.412 & 8.65 & 11.02 & 14.90 & 20.15 & 25.75 & 9.13 & 17.10 & 6.25 & 10.85 & 1.736 \\
\hline 17.0 & 0.392 & 9.83 & 12.38 & 16.50 & 22.00 & 27.78 & 9.62 & 17.95 & 6.67 & 11.28 & 1.691 \\
\hline 20.0 & 0.361 & 12.07 & 14.96 & 19.50 & 25.42 & 31.53 & 10.46 & 19.46 & 7.43 & 12.03 & 1.619 \\
\hline 24.9 & 0.323 & 15.86 & 19.24 & 24.40 & 30.95 & 37.56 & 11.71 & 21.70 & 8.54 & 13.16 & 1.541 \\
\hline 27.7 & 0.306 & 18.07 & 21.71 & 27.20 & 34.08 & 40.95 & 12.37 & 22.88 & 9.13 & 13.75 & 1.506 \\
\hline 32.0 & 0.285 & 21.53 & 25.54 & 31.50 & 38.85 & 46.10 & 13.31 & 24.57 & 9.97 & 14.60 & 1.464 \\
\hline
\end{tabular}

Note that the appearance of the peak $\left(t_{\mathrm{p}}^{*}\right)$ is $0.5 \mathrm{~s}$ earlier than the spatial $t_{\mathrm{p}}$.

\subsection{The sample size effect}

It is common to use larger injection size in FIA (compared to chromatography) to avoid losing sensitivity. Therefore the injection volume cannot be treated like a small plug. Instead, the sample section should be regarded to have a histogram-shape at the beginning; which gradually becomes a plateau, then a peak shape when $t_{\mathrm{p}}^{*}$ becomes large. Taking the present case for instance, the injection volume was $0.357 \mathrm{ml}$, at a flow-rate of $4.25 \mathrm{ml} / \mathrm{min}$ it equals an initial zone width $\left(W_{i}\right)$ of ca. $5 \mathrm{~s}$ on the time scale.

Accordingly, the TCG function is derelict within $10 \mathrm{~s}$ due to the large sample size. For example, the initial absorbance of the dye was 0.805 ; but the TCG simulation gives infinite $h$ when $t_{\mathrm{p}}$ is zero (Fig. 7b). Also, experimentally the initial width components $a$ and $b$ were both $2.5 \mathrm{~s}$, leading to an initial $A_{f}=1$, while the TCG gives infinite $A_{f}$ at $t_{\mathrm{p}}^{*}=0$ because the initial band width is assumed zero (Fig. 7c). The difference on the peak shapes in the first 10s (or $L_{c}<20 \mathrm{~cm}$ ) is also noticeable. Experimental peaks are fatter at the middle, whereas simulated peaks have much slender looks even though the bottom $\left(W_{0.1}\right)$ is wider.

\subsection{Comparison on asymmetry factor}

Further comparison was done on peaks with $t_{\mathrm{p}}^{*}$ larger than $10 \mathrm{~s}\left(t_{\mathrm{p}}^{*} / w_{i}>2\right)$ to skip the sample size effect. Although the digitizing of the experimental peak widths might not be very precise, it does give clear trends as can be seen in Fig. 7. Both $a$ and $b$ components gradually merge with the theoretical lines when $t_{\mathrm{p}}^{*}$ is larger than $15 \mathrm{~s}$. Within the range of $10-20 \mathrm{~s}$ the asymmetry factor $\left(A_{f}=b / a\right)$ for the experiment peak is slight larger than of the TCG simulation. Therefore, the temporal effect may not be, although dominant, the only one that controls the peak tailing.

The contributions by the temporal effect have been estimated by taking $\left(A_{f}-1\right)$ as a parameter (Fig. $7 d$ ). For peaks with $t_{\mathrm{p}}^{*}$ between 15 and $20 \mathrm{~s}\left(t_{\mathrm{p}}^{*} / W_{i}=3-\right.$ $4)$, the temporal effect conveys a contribution of $>90 \%$ for the experimental deformation, leaving less than $10 \%$ for other effects. When $t_{\mathrm{p}}^{*}$ is above $25 \mathrm{~s}$ $\left(t_{\mathrm{p}}^{*} / W_{i}>5\right)$, the temporal contribution raises in proportion to nearly $100 \%$, but reduces in scale.

\subsection{A general aspect for FIA peaks}

Based on the above verifications, a general aspect for the FIA peak shape development stands out and it can be stated in four stages:

Stage 1: At the initial stage or when $t_{\mathrm{p}}^{*}$ is close to the initial zone width $W_{i}\left(t_{\mathrm{p}}^{*} / W_{i}<2\right.$ in the present work), the sample size effect is the major concern, 
(a)

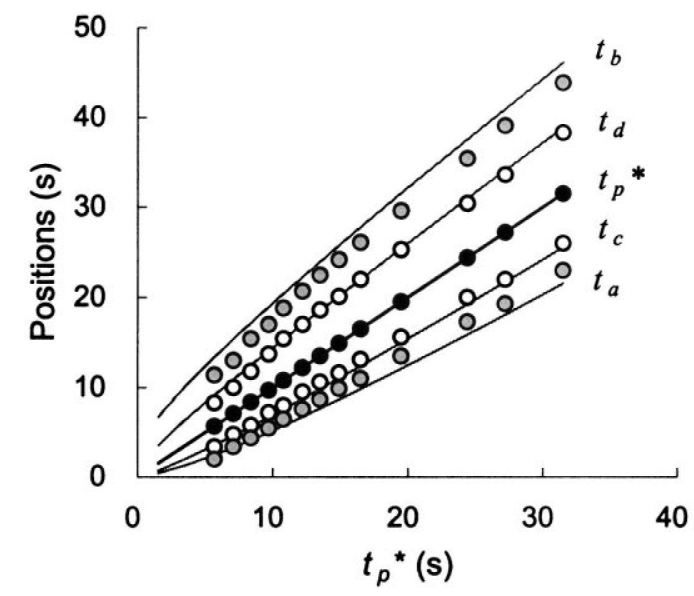

(c)

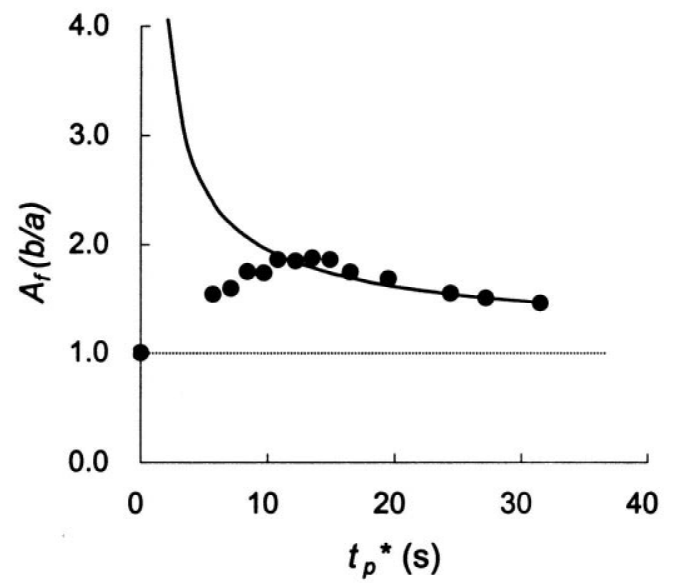

(b)

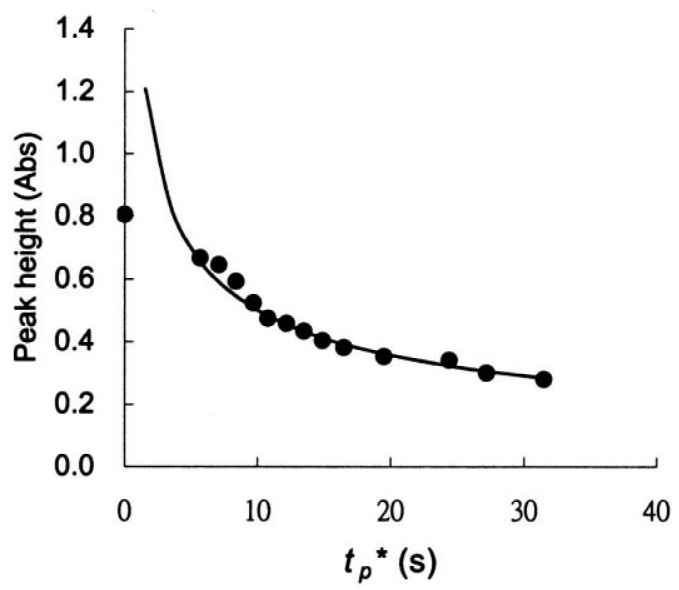

(d)

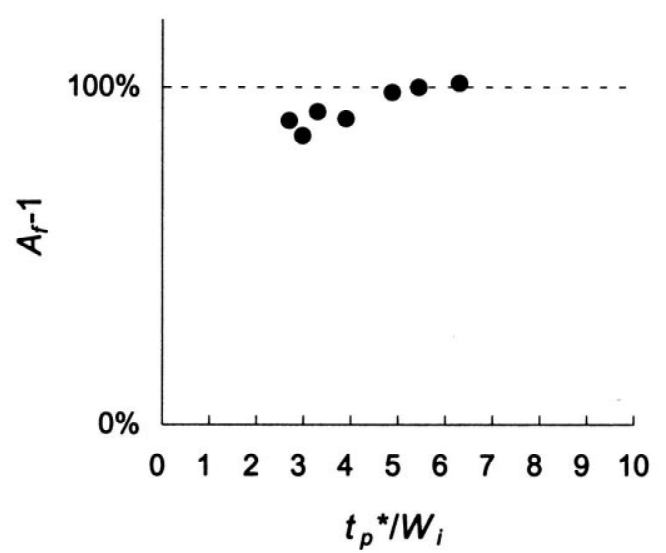

Fig. 7. Comparison of parameters estimated from the observed peaks (circles) and from that generated by TCG equation (lines). (a) Position of $t_{a}, t_{b}, t_{c}, t_{d}$ versus $t_{\mathrm{p}}^{*}$; (b) peak height versus $t_{\mathrm{p}}^{*}$; (c) asymmetric factor, $A_{f}=b / a$, versus $t_{\mathrm{p}}^{*}$; (d) temporal contribution $\left(A_{f}-1\right)$ versus $t_{\mathrm{p}}^{*} / W_{i}$.

which gives a plateau-shape pattern and masks all other effects.

Stage 2: When $t_{\mathrm{p}}^{*}$ is larger (e.g., $t_{\mathrm{p}}^{*} / W_{i}>2$ in this work), the initial sample zone becomes a peak, appearing with an asymmetrical tail. Both spatial (e.g., the sample size effect and the Poisseulie effect) and temporal components may co-exist and co-responsible for the peak skewness.

Stage 3: When $t_{\mathrm{p}}^{*}$ is even larger $\left(t_{\mathrm{p}}^{*} / W_{i}>5\right.$ in this work), the spatial effects fade gradually leading to a Gaussian-like distribution in the tubular channel, but it is still viewed to have a tail on the recorder chart. The temporal effect becomes the dominating reason for the peak deformation.

Stage 4: When $t_{\mathrm{p}}^{*}$ is very large (say, $t_{\mathrm{p}}^{*} / W_{i}>50$ ), the asymmetry factor $A_{f}$ converges to 1 and the peak appearance becomes a Gaussian curve. Neither the spatial nor temporal effect is significant, the observed peak shape reveals almost the same pattern as the mass profile.

In FIA application, it is quite common to have an injection size of $0.1-0.5 \mathrm{ml}$ and a residence time of 
$30 \mathrm{~s}-2 \mathrm{~min}$ for a sample peak. If the manifold is operated at a flow-rate of $5 \mathrm{ml} / \mathrm{min}$, the $t_{\mathrm{p}}^{*} / W_{i}$ ratios lay in a range between 5 and 50 (i.e., stage 3). With this in mind one may consider that most of the peak skewness observed for FIA should be regarded as temporal. For chromatography the $t_{\mathrm{p}}^{*} / W_{i}$ is usually very large $(>100)$, the temporal peak tailing can only be observed for those leading peaks at near the void position.

\section{Conclusion}

There are no contradictions between the proposed TCG equation and existing theories for chromatography and for FIA, as all lead to the same conclusion: the peak is skewed when time is short and gradually turns to a Gaussian-like curve when the time is prolonged. The difference would be that the present work "emphasizes" the temporal effect, which transforms the mass distribution profile from a spatial axis to a sequential time axis and gives a twisted peak shape on the recorder. It provides clues for those who are not familiar enough with physical or mathematical skills to understand when and why is the peak not symmetrical.

The temporal effect exists universally in either FIA or chromatography. However, it seems that many scientists of both fields have neglected this effect completely, leading to contradictions when they need to explain "unexpected" peak tailings: chromatographers tend to explain in a kinetic way, whereas FIA users tend to elucidate on the Poisseulie effect. This could have been arisen from the recognition of the theoretical plate numbers. For example, FIA may have apparent smaller plate numbers due to the lacking of stationary phase and faster flow-rate. However, it should be borne in mind that the scaling is artificially defined, therefore direct comparison on plate numbers might not be appropriate.

The use of "time" as a parameter to evaluate the zone broadening is deemed more practical. Any disparity from the TCG curve could account for the contribution other than the temporal effect.

\section{Acknowledgements}

The author would like to thank K.M. Chen, Y.H. Li, G.T.F. Wong, K. Ronning, and two anonymous reviewers for their kind and useful criticism of the manuscript.

\section{References}

[1] J. Růžička, E.H. Hansen, in: Flow Injection Analysis, 2nd ed, Wiley, New York, Chichester, Brisbane, Toronto, Singapore, 1988.

[2] J. Růžička, G.D. Christian, Analyst 115 (1990) 475.

[3] L.C. Craig, J. Biol. Chem. 155 (1944) 519.

[4] A.J.P. Martin, R.L.M. Synge, Biochem. J. 35 (1941) 1358.

[5] J.C. Giddings, H. Eyring, J. Phys. Chem. 59 (1955) 416.

[6] L. Lapidus, N.L. Amundson, J. Phys. Chem. 56 (1952) 984.

[7] G. Carta, Chem. Eng. Sci. 43 (1988) 2877.

[8] J.B. Rosen, J. Chem. Phys. 20 (1952) 387.

[9] S. Golshan-Shirazi, G. Guiochon, in: F. Dondi, G. Guiochon (Eds.), Theoretical Advancement in Chromatography and Related Separation Technqiues, Kluwer, Dordrecht, Boston, London, 1991.

[10] K. Robards, P.R. Haddad, P.E. Jackson, in: Principles and Practice of Modern Chromatographic Methods, Academic Press, London, Boston, San Diego, New York, Toronto, Sydney, Tokyo, 1994.

[11] G. Taylor, Proc. R. Soc. London, Ser. A 219 (1953) 186.

[12] R. Tijssen, Anal. Chim. Acta 114 (1980) 71.

[13] J.M. Reijn, W.E. van der Linden, H. Poppe, Anal. Chim. Acta 114 (1980) 105.

[14] J.T. Vanderslice, K.K. Stewart, A.G. Rosenfeld, D.J. Higgs, Talanta 28 (1981) 11.

[15] S.H. Isaac, H. Soeber, L.H. Cristensen, J. Villadsen, Chem. Eng. Sci. 47 (1992) 1591.

[16] D.A. Skoog, F.J. Holler, T.A. Nieman, in: Principles of Instrumental Analysis, 5th ed, Saunders College Pub./Harcourt Brace College Publishers, Philadelphia/Orlando, 1998.

[17] E. Glueckauf, Trans. Faraday Soc. 51 (1955) 34.

[18] R. de Levie, in: Principles of Quantitative Chemical Analysis, McGraw-Hill, International Editions, New York, 1997.

[19] J.P. Foley, J.G. Dorsey, J. Chromatogr. Sci. 22 (1984) 40.

[20] D. Hanggi, P. Carr, Anal. Chem. 57 (1985) 2394.

[21] J.P. Foley, J.G. Dorsey, Anal. Chem. 55 (1983) 730.

[22] J.P. Foley, Anal. Chem. 59 (1987) 1984.

[23] D.C. Harris, in: Quantitative Chemical Analysis, 4th ed, W.H. Freeman, San Francisco, CA, 1995.

[24] R. Aris, Chem. Eng. Sci. 9 (1959) 266.

[25] B.A. Bidingmeyer, F.V. Warren Jr., Anal. Chem. 56 (14) (1984) 1583A. 